\title{
Morphology Element Research on Chinese Small-Sized Liquor Bottle Design
}

\author{
Ye Zhang ${ }^{1,2}$, Huanzhi Lou ${ }^{1}$, Hui Yu ${ }^{2}$ \\ ${ }^{1}$ School of Architecture and Art Design, \\ Beijing Jiaotong University, \\ Beijing 100044, China \\ 531892226@qq.com
${ }^{2}$ School of Creative Technologies, University of Portsmouth
Portsmouth PO1 2DJ, United Kingdom
hui.yu@port.ac.uk

\begin{abstract}
Based on Kansei engineering methods and performed user testing, this work analyzes small-sized liquor bottle of the Chinese liquor market. Firstly, perform the cluster analysis and multidimensional scaling (MDS) analysis to obtain 26 representative sample bottles. Secondly, According to the analysis results of an expert group, the shape of the bottle is divided into six feature categories and 36 morphological unit categories. And then, the factor analysis table is built according to sample bottles. After that, the level of user preferences on bottle shape is evaluated by the image scale method over a number of consumers. By investigating associations between the user preference values and the form elements table from the values of the statistics table, researchers have found a preliminary result that some elemental characteristics indeed have a greater impact on consumer preferences. Finally, Pearson product-moment correlation coefficient inspection method is used to verify the conclusion mentioned above. Based on the correlation some elements are the key factors that affect consumer preferences in Chinese liquor market, while some are not preferable elements by customers. Those non-preferable elements should be considered to avoid in design. This study provides important reference and theoretical support for Chinese liquor bottle design and brand development.
\end{abstract}

Keywords-component; formatting; style; styling; insert (key words)

\section{INTRODUCTION}

Liquor is one of the most important categories in food industry. With the development of Chinese economy in recent years and changes in social values, the traditional attribution of liquor as gift value is replaced by form of ownership. Due to poor sales in the $1 \mathrm{~kg}$ Pack liquor gifts in recent years, the survival pressure of liquor enterprises makes them focus more on young consumer groups. They investigated the status of liquor market, and then, started to promote $100-250 \mathrm{ML}$ products. Since 2012, all major companies rolled out over more than 200 small-sized liquor brands. Small-sized liquor will be an important development direction of liquor products and brands in the future. The shape design of liquor bottle is one of the most crucial and urgent problems. The product image plays an important role in the consumer's preference and choice of the products [1]. An appropriate bottle design can effectively deepen people's impressions of the product, and thereby enhance the consumer's desire to buy the product. Consumer-oriented Kansei Engineering has been developed as a methodology that transforms a consumer's feeling or image about a product into the design elements of the products [2]. With Kansei research method, the proposed research attempts to look for the association rules between bottle-type form characteristic elements and consumer preferences.

Based on the principles of consumer-oriented, first the consumer's interests are tested in bottle-type and willingness of purchase under the same conditions. Second, further analysis is taken on liquor bottle design and classifies language elements from common design patterns with expert group. Third, Pearson productmoment correlation coefficient is used to study the relevancy between the form factor and user preferences. Finally, this paper describes the research process and methods in detail.

\section{USER TESTING}

In this research, the methodology is based on Kansei Engineering. Kansei Engineering has been applied successfully in the product design field in order to explore the relationship between the feeling (perception of the product image) of the consumers and the design elements of the product [3-7]. Perceptual studies process from collection to analysis is divided into four stages. Firstly, three groups of test subjects are establihed. Personnel of each testing group cooperate according to their functions. Second, the final testing samples are established by market survey group and the Panel of experts. Third, the Panel splits the samples and product elements table. Fourth, an online questionnaire of consumer preferences is achieved through the scale method. By the most suitable adjective pairs, Intention multidimensional method is used to quantize the level of users' psychological preference, combining with the design result of pictures [8]. Finally, we analyse user preference testing data obtained previously using the Pearson product moment correlation coefficient and decide the key issues to the design of small-sized liquor regarding to morphological elements. The details of each step are described in the rest of this paper.

\section{A. Establish experimental group}

Participants in the study are mainly divided into three parts. Group-A has ten people, which are seven men 
and three women, with average age of 27.4 years old. They have drinking experience, but not alcoholics. They are assigned to collect $100-250 \mathrm{ml}$ small-sized bottles that existed in the market. They need to visit various shopping malls and buy as many samples of goods as possible. And they will remove the outer packages and logos of the bottle body pattern information of these samples and label them uniformly in order to prevent the final styling elements from the impact of graphic design, brand, color, and other non-bottle-type interference in perceptual studies process. Group-B is the expert group of five members with average age of 35.5 years old. There are two men and three women in this group. They have six years' experience in product design and brand planning, and they will classify main elements of key morphological characteristics of the typical liquor bottle based on experience and morphological analysis. Group discussion is used to determine the final 26 pieces of representative pictures for user testing (Figure 1). Group$\mathrm{C}$ consists of the subjects of perceptual studies. This research uses a network data collection with a total number of 90 copies of questionnaires issued. The number of effective feedback copies is 79 with $88 \%$ valid rate. The age of subjects ranges from 18 to 55 years old. Male $48.15 \%$, female $51.85 \%, 18-23$ years old people accounted for $25.93 \%, 23-28$ years old subjects accounted for $39.51 \%, 28-33$ years old participants accounted for $17.28 \%$, and only $1.23 \%$ for the age over 38. According to the feedbacks, we can conclude that the subject can represent the new generation, and they have drinking experiences, but are not alcoholics. By using the

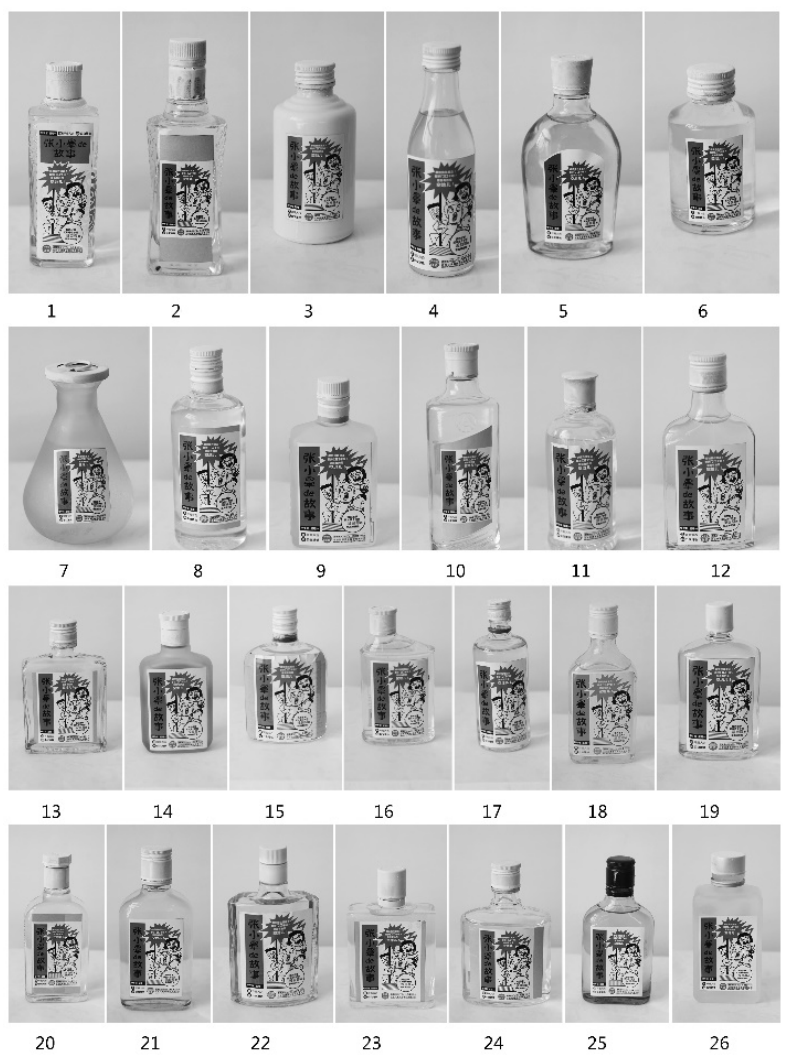

Fig.1 26 Test samples
SD method over these subjects, we can achieve the bottle intent scale scores, and get the statistical data with respect to test scores of 79 valid subjects over 26 sample bottles. After calculating the mean value and the standard deviation, these values can be seen as one of the basic data source for further analysis of user intentions.

\section{B. Image Samples to Determine}

This study is aimed at small-sized alcoholic commodity whose volume of bottles is $100 \mathrm{ml}$ to $250 \mathrm{ml}$. The market research staff (Group-B) visited all large, medium and small-sized markets and collected 65 samples with different brand and styles. These products are available to market after 2013 . They can represent the majority of small-sized alcoholic commodities in current market. After collecting enough samples, without any pattern information such as packages and logos on the bottle body, we use uniform consistent external stickers and take pictures with white background (Fig.1). Through a computer screen with Kawakida Jirou Method ${ }^{[8]}$ based on similarity, the samples are divided into different groups. Then, based on the method of multiple scales (MDS) population analysis [9], 26 representative products are obtained finally. Then according to 26 of the elected sample, 5 members of the Group of experts produced morphological analysis tables.

\section{Form Factors division}

According to the 26 representative samples, based on morphological analysis, panelists (Group-B) describe the form elements of the samples based on their own experience and domains. First, they induce constituent elements of the bottle through several revisions and reviews. They delete duplicate and non-essential factor and retain important components of the bottles. Based on the characteristics of the product form, a bottle consists of four parts: caps, bottleneck, bottle shoulder, and bottle body. The different parts are subdivided into their morphological components, which follows the principle of non-redundant and no shortage of items. The sample is divided into six morphological characteristic categories (Table 1), where caps, based on the ratio of the width and height, can be divided into four classes. There are six kinds of forms in the shape of the bottle cap. Bottlenecks can be divided into five categories. Bottle shoulder can be divided into eight categories. Based on the scale of the bottle body, it can be divided into seven categories. According to the top view, five most important features can be extracted. Then, the panel will be matched the 39 kinds of form feature elements to the 26 test bottle samples. Each bottle has its own shape feature element code. For example, as for the sample No. 8, the representative elements are $\mathrm{X} 1=4, \mathrm{X} 2=2, \mathrm{X} 3=4, \mathrm{X} 4=$ $3, \mathrm{X} 5=4, \mathrm{X} 6=2$. These form the important element data for further analysis of the feature elements of the bottles. 


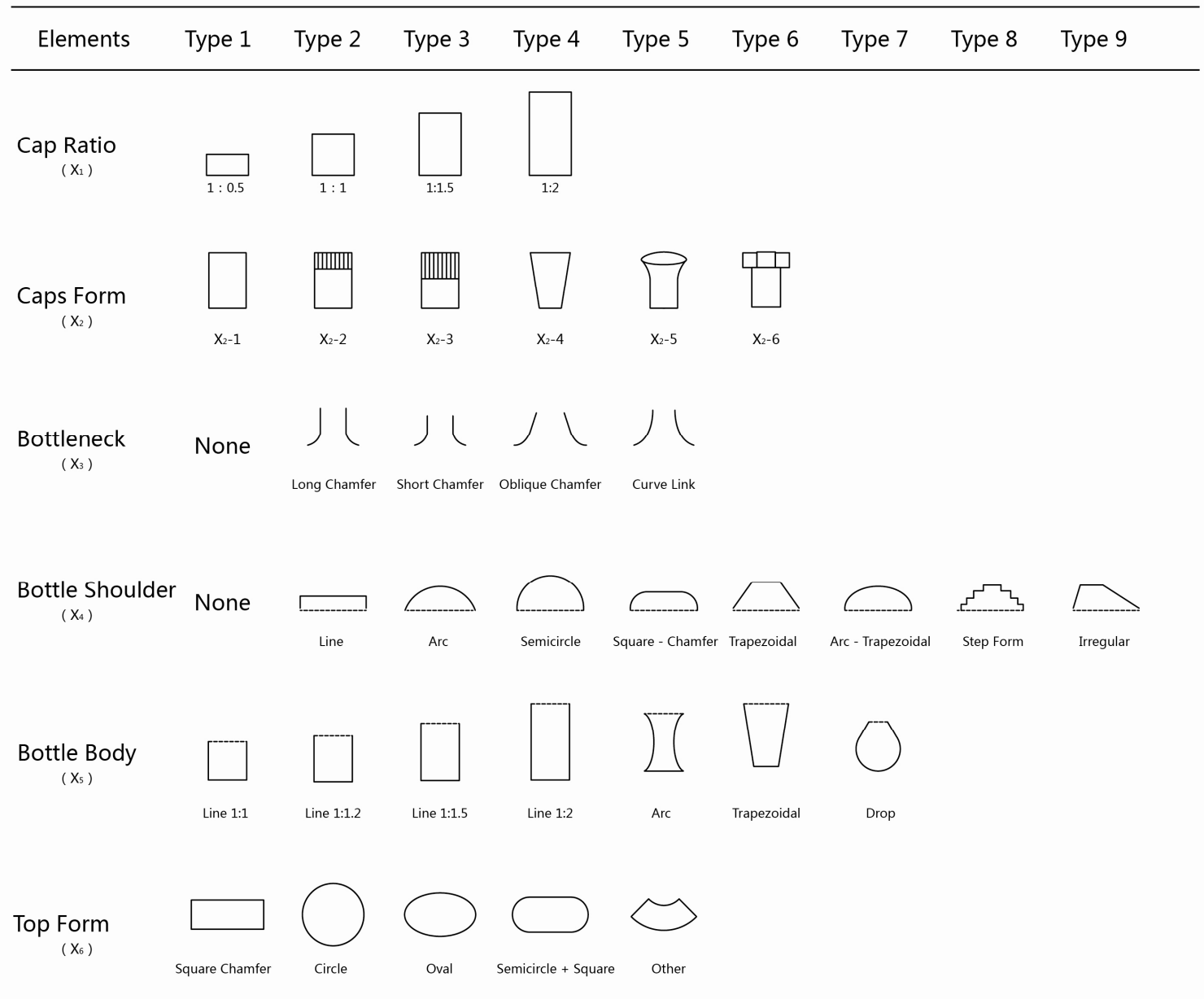

\section{Investigation of Consumer Preferences}

The research process uses an intuitive way. First, it assumes that all samples are in the same condition to assess the degree of consumer preference for a product shape and willingness to purchase. We use the 7 -scores intention scales with score one meaning absolutely dislike and seven as a favorite. Dislike-Like (D-L) is used to indicate the degree of user preferences. In the same way not want to buy-Want to buy $(\mathrm{N}-\mathrm{W})$ is used to indicate the degree of expectation to purchase. Subjects are asked to fill the form with the score of their feelings to the 26 sample. And then, we compute mean and standard deviation values according to the statistics. After discussion from the panel of experts, we use the first set of questions (D-L) for study. In everyday life, the degree of preference to a product deeply influences the willingness to purchase it, and also can be seen as an important reference to explore what product form factors are more important to consumer preferences. Table 2 shows that the first line is the column name, $\mathrm{X} 1$ to $\mathrm{X} 6$ are morphological characteristic symbols. Line 3 to 28 show the 26 sample elemental codes, the degree of preferences, standard deviation and the mean values of expectation purchase.

\section{E. Preliminary Analysis}

Through a questionnaire investigation, value of the consumer preferences is got for each product. It can be seen from the results that the highest average value is 4.24 from the sample No.7. The second is No.9, whose average is 3.987. The lowest average value is 2.924 of the samples No.15, and the second lowest average value is 2.937 of No.24. By comparative analysis of the sample scores sorting and distribution of elements types, following rules can be taken. 


\begin{tabular}{|c|c|c|c|c|c|c|c|c|c|}
\hline \multirow[t]{2}{*}{ Sample no. } & \multirow[t]{2}{*}{$\mathrm{X} 1$} & \multirow[t]{2}{*}{$\mathrm{X} 2$} & \multirow[t]{2}{*}{$\mathrm{X} 3$} & \multirow[t]{2}{*}{$\mathrm{X} 4$} & \multirow[t]{2}{*}{$\mathrm{X} 5$} & \multirow[t]{2}{*}{$\mathrm{X} 6$} & \multicolumn{2}{|c|}{ D-L value } & \multirow{2}{*}{$\begin{array}{c}\mathrm{N}-\mathrm{I} \\
\text { Average }\end{array}$} \\
\hline & & & & & & & Average & Standard deviation & \\
\hline 1 & 3 & 2 & 4 & 2 & 6 & 1 & 3.684 & 2.066 & 3.506 \\
\hline 2 & 4 & 3 & 4 & 2 & 5 & 2 & 3.620 & 2.096 & 3.430 \\
\hline 3 & 1 & 2 & 3 & 8 & 3 & 2 & 3.051 & 1.954 & 2.873 \\
\hline 4 & 2 & 2 & 5 & 1 & 4 & 2 & 3.418 & 2.048 & 3.342 \\
\hline 5 & 3 & 4 & 2 & 4 & 6 & 3 & 3.684 & 1.885 & 3.430 \\
\hline 6 & 1 & 2 & 1 & 6 & 3 & 2 & 3.532 & 1.973 & 3.380 \\
\hline 7 & 1 & 1 & 2 & 1 & 7 & 2 & 4.241 & 2.260 & 4.038 \\
\hline 8 & 4 & 2 & 4 & 3 & 4 & 2 & 3.266 & 1.708 & 3.101 \\
\hline 9 & 3 & 3 & 1 & 6 & 2 & 1 & 3.987 & 2.016 & 3.873 \\
\hline 10 & 3 & 2 & 2 & 3 & 4 & 4 & 3.443 & 1.824 & 3.342 \\
\hline 11 & 2 & 5 & 3 & 5 & 3 & 2 & 3.481 & 1.846 & 3.405 \\
\hline 12 & 2 & 2 & 2 & 7 & 2 & 1 & 3.532 & 1.920 & 3.316 \\
\hline 13 & 2 & 2 & 3 & 6 & 1 & 1 & 2.987 & 1.891 & 2.962 \\
\hline 14 & 3 & 2 & 3 & 3 & 2 & 1 & 3.608 & 1.793 & 3.557 \\
\hline 15 & 3 & 2 & 3 & 3 & 1 & 3 & 2.924 & 1.810 & 2.810 \\
\hline 16 & 2 & 2 & 1 & 9 & 2 & 4 & 3.076 & 2.093 & 2.975 \\
\hline 17 & 3 & 2 & 3 & 3 & 4 & 2 & 3.241 & 1.726 & 3.203 \\
\hline 18 & 3 & 2 & 5 & 7 & 3 & 4 & 3.291 & 1.848 & 3.000 \\
\hline 19 & 2 & 1 & 3 & 3 & 2 & 3 & 3.633 & 1.988 & 3.443 \\
\hline 20 & 3 & 6 & 3 & 4 & 2 & 1 & 3.722 & 1.881 & 3.481 \\
\hline 21 & 3 & 2 & 3 & 7 & 3 & 1 & 3.684 & 1.871 & 3.456 \\
\hline 22 & 3 & 3 & 2 & 2 & 2 & 5 & 3.013 & 1.829 & 2.924 \\
\hline 23 & 3 & 1 & 1 & 2 & 1 & 1 & 3.266 & 1.886 & 3.114 \\
\hline 24 & 2 & 2 & 4 & 3 & 1 & 3 & 2.937 & 1.727 & 2.785 \\
\hline 25 & 3 & 2 & 4 & 7 & 2 & 4 & 3.506 & 2.075 & 3.392 \\
\hline 26 & 2 & 1 & 3 & 6 & 3 & 1 & 3.937 & 2.090 & 3.722 \\
\hline
\end{tabular}

- Cap ratio is not the key factor that determines consumer preference.

- Cap form greatly affects the consumers ' preferen ces. Obviously, consumers dislike X2 whose top half is a short knob. However, this kind of cap is the mainstream product in the market.

- In a whole view, the bottleneck cannot attract consumer concerns, but what is worth of attention is that X3-5 bottlenecks get negative feedback from consumers. Perhaps this is where the designers should note.

- Bottle shoulder is an important part of the bottles. From data analysis, we found that the 7th sample without bottle shoulder get the highest score. In the case where the samples have the bottle shoulder, the statistics show that consumers dislike the asymmetry bottle shoulder. They prefer Semicircle (X4-4) and arc trapezoid (X47), but dislike have a curved shape (X4-3) of the bottle.
- Bottle body is the largest and most important visual component. It is clear from the data. We found that consumers prefer those bottle bodies whose ratio between length and width is $1: 1.2$ (X5-2). It can be seen through the analysis that consumers dislike 1:1 square bottle (X5-1) and 1:2 lanky linear bottle (X5-4). Consumers are not concerned about whether the bottles are straight or have some curvatures. In addition, the teardrop-shaped (X5-7) bottle gets the highest score. Such bottle is so rare on the market that we only found one sample. Researchers are not optimistic about such teardrop-shaped bottle. Because of the too small sample results, it cannot come to a conclusion. But with the results of consumer testing, more research is needed to understand the value of such bottle shape.

- Top view features determine the sense of thickness of a bottle, and reflects overall relationship between the front and side outline. As is obviously shown from the data results, 
consumer prefer squared bottle with a small-sized fillet (X6-1), rather than a straight line + semicircle type (X6-4). For cylindrical, oval cylindrical ones, consumers express that it does not matter whatever. This shows that the current consumers prefer clean, simple designs with some details of lines, and like conventional flatsquare bottle most.

Our aim is to further analyze several relationships based on Pearson product moment.

\section{PEARSON CORRELATION COEFFICIENT ANALYSIS}

Using Pearson correlation coefficient analysis, the relationship between the presented bottle styles and subject ratings has been computed by the following equation:

The proportion of the element styles was taken where the correlation was statistically significant at the significance level $p<0.05$. Fig.2(a) and (b) demonstrates the statistical significance rating number of the $1^{\text {st }}$ and $7^{\text {th }}$ level rating respectively.

$$
r_{x y}=\frac{n \sum_{i=1}^{n} x_{i} y_{i}-\sum_{i=1}^{n} x_{i} \sum_{i=1}^{n} y_{i}}{\sqrt{n \sum_{i=1}^{n} x_{i}^{2}-\left(\sum_{i=1}^{n} x_{i}\right)^{2}} \sqrt{n \sum_{i=1}^{n} y_{i}^{2}-\left(\sum_{i=1}^{n} y_{i}\right)^{2}}}
$$

In general, the Pearson correlation coefficient analysis has shown the significant styles correlated with consumers' ratings. The style of each element can be obtained according to those coefficients. As it can be seen from Fig. 2, there are clearly different subcategories
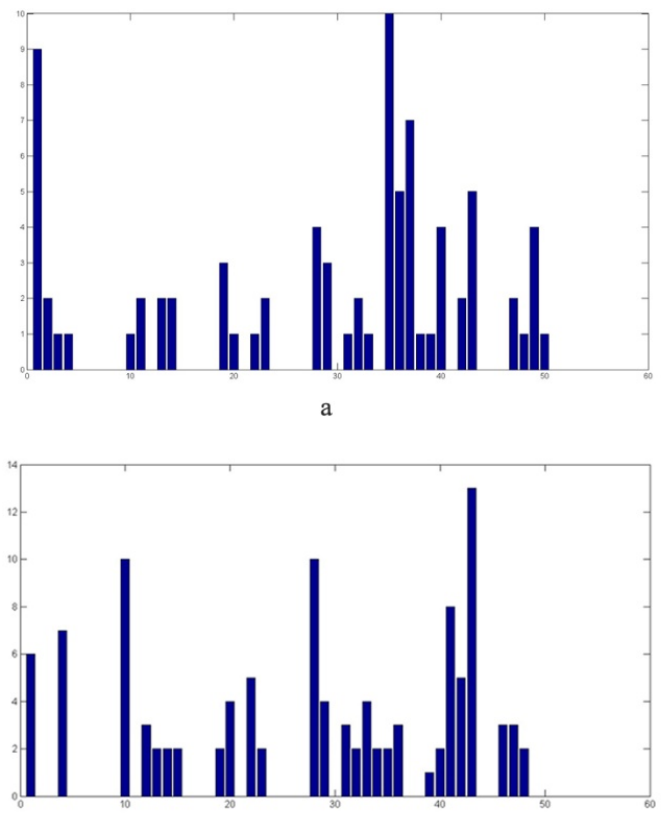

b

Fig. 2. Statistically significant design style (a) statistical significance rating number of the $1^{\text {st }}$ level rating. (b) Statistical significance rating number of the $7^{\text {th }}$ level rating rated as the significant design styles for these two rating levels.
The $\mathrm{x}$-axis represents the subcategories of the design style and the $y$-axis is the significance rating number. In Fig. 2 (a), there are a few subcategories rated above 4, which are taken as the most related design styles based on the 26 given images. Fig. 2 (b) clearly illustrates the significant styles for each element.

\section{CONCLUSION}

Currently, Chinese small-sized liquor market gradually booming. Using which bottle shape is a key issue that troubled companies and designers. Based on the test of consumer psychology preferences and Kansei engineering methods, this research focuses on the smallsized liquor bottle shape and design elements. The study comes into final conclusion:

1) Statistical analysis based on subject's rating can provide clues of important elements and styles in liquor bottle design. For example, bottle shoulder is an important design element while the ratio 1:1.2 (X5-2) for the length to width of the bottle body is preferable from the survey data.

2) Pearson correlation coefficient between the subject rating and sample style data can clearly show the preferable design styles for each element of the bottle. This provides the designer the significant design style from the point of view of subject.

These conclusions will provide an important reference in the development of Chinese liquor bottle design, especially in the choice of morphological language elements. In the future, more examinations on some other components will been taken that may affect consumer preferences, such as labels shapes, brands, words, illustrations, color and so on. Therefore, based on consumer preferences, a complete system can be built up for the research of visual design strategy of small-sized liquor. This method will provide important theoretical support for bottle product.

\section{ACKNOWLEDGMENT}

This project was supported by "the Fundamental Research Funds for the Central Universities" (2014JBW001, Beijing Jiaotong University, China). The authors also thank all the subjects in china for their participation and assistance in the experimental study.

\section{REFERENCES}

[1] Chuang. MC, Chang. CC, Hsu. SH, "Perceptual elements underlying user preferences toward product form of mobile phones," International Journal of Industrial Ergonomics, vol.27, pp. 247-258, 2001.

[2] Nagamachi.M., "Kansei engineering: a new ergonomics consumeroriented technology for product development," International Journal of Industrial Ergonomics , vol.15, pp. 3-11,1995.

[3] Ishihara. S, Ishihara. K, Nagamachi. M, "An automatic builder for a kansei engineering expert system using selforganizing neural net- works," International Journal of Industrial Ergonomics, vol. 15, pp. 25-37,1995.

[4] Lin. YC, Lai, . HH, Yeh. CH, "Consumer-oriented product form design based on fuzzy logic: a case study of mobile phones," International Journal of Industrial Ergonomics , vol.37, pp. 531-543,2007. 
[5] Schutte. S, Eklund. J, "Design of rocker switches for workvehicles-an application of Kansei Engineering," Applied Ergonomics, vol. 36, pp. 557-567,2005.

[6] Zhai. LY, Khoo. LP, Zhong. ZW, "A rough set based decision support approach to improving consumer affective satisfaction in product design," International Journal of Industrial Ergonomics, vol. 39, pp. 295-302, 2009.

[7] Yang-Cheng Lin, Chung-Hsing Yeh, Chun-Chun Wei, "How will the use of graphics affect visual aesthetics? A user-centered approach for web page design," International Journal of Human-Computer Studies , vol. 71, pp. 217227,2013

[8] Cross.M, Engineering Design Methods. Wiley, London.Deng, J.-L, "Control problems of grey system," System and Control Letters, vol. 1, pp. 288-294,1994.

[9] Hair. J, Anderson. R,Tatham. R, Black. W,"Multivariate Data Analysis," New York:Macmillan Publishing, 1995. 